\title{
Vacinação: do senso comum à consolidação do conhecimento científico
}

\author{
Vaccination: from common sense to the consolidation of scientific knowledge \\ Vacunación: del sentido común a la consolidación del conocimiento científico
}

Carolina Fachetti Loyola ${ }^{1}$, Gabriela Freitas da Silveira ${ }^{1}$, Isabella Colicchio de Paula Costa ${ }^{1}$, Laura Finotti Frausino ${ }^{1}$, Laura Rohlfs Taquary ${ }^{1}$, Rhaísa Ghannam Macedo ${ }^{1 *}$.

\section{RESUMO}

Objetivo: Descrever o conhecimento de pacientes renais crônicos a respeito do Calendário Nacional de Imunização, assim como o cumprimento do processo vacinal. Métodos: Trata-se de um estudo transversal descritivo de abordagem quantitativa, conduzidos com 115 pacientes renais crônicos, com idade superior a 18 anos. Os dados foram coletados por meio de questionários transferidos para planilhas no Microsoft Excel Office, onde foram tabulados e descritos em forma de tabelas e gráficos. Posteriormente, foram analisados por estatística descritiva com auxílio do software IBM SPSS Statistics. O estudo foi aprovado por Comitê de Ética em Pesquisa. Resultados: A amostra, com média de idade de 55,5 $\pm 5,0$ anos (27\%), apresentou maioria masculina $(58,3 \%)$; com o ensino fundamental completo $(37,4 \%)$; renda familiar média, de até 1 salário-mínimo (44,3\%). Destes, apenas $41,7 \%$ dos indivíduos foram alertados sobre a importância do calendário vacinal. Além disso, 86,1\% alegaram acreditar que as vacinas fazem bem, em contradição aos $90,4 \%$ que mostraram cartões de vacina desatualizados. Conclusão: Conclui-se que existe um largo espaço entre o ideal e o real, no que tange às práticas de saúde para fins de vacinação e cumprimento assíduo do cartão vacinal, enfatizando sua importância e a problemática relacionada a ausência dessa prevenção.

Palavras-chave: Vacinação, Programas de imunização, Cobertura vacinal.

\begin{abstract}
Objective: Describe the knowledge of chronic kidney patients regarding the National Immunization Calendar, as well as compliance with the vaccination process. Methods: This is a descriptive cross-sectional study with a quantitative approach, conducted with 115 chronic renal patients, aged over 18 years. Data were collected through questionnaires transferred to spreadsheets in Microsoft Excel Office, where they were tabulated and described in the form of tables and graphs. Subsequently, they were analyzed using descriptive statistics with the help of the IBM SPSS Statistics software. The study was approved by the Research Ethics Committee. Results: The sample, with a mean age of $55.5 \pm 5.0$ years $(27 \%)$, had a male majority ( $58.3 \%)$; with complete primary education (37.4\%); average family income of up to 1 minimum wage (44.3\%). Of these, only $41.7 \%$ of individuals were aware of the importance of the vaccination schedule. In addition, $86.1 \%$ claimed to believe that vaccines are good, in contrast to $90.4 \%$ who showed outdated vaccine cards. Conclusion: It is concluded that there is a wide space between the ideal and the real, regarding health practices for vaccination purposes and assiduous compliance with the vaccination card, emphasizing its importance and the problems related to the absence of this prevention.
\end{abstract}

Keywords: Vaccination, Immunization program, Vaccination coverage.

\footnotetext{
1 Universidade Evangélica de Goiás (UniEVANGÉLICA), Anápolis - GO.

*E-mail: mailto:rhaisamacedo_@hotmail.com
} 


\section{RESUMEN}

Objetivo: Describir el conocimiento de los pacientes renales crónicos sobre el Calendario Nacional de Inmunizaciones, así como el cumplimiento del proceso de vacunación. Métodos: se trata de un estudio descriptivo transversal con abordaje cuantitativo, realizado con 115 pacientes renales crónicos, mayores de 18 años. Los datos se recolectaron a través de cuestionarios transferidos a hojas de cálculo en Microsoft Excel Office, donde se tabularon y describieron en forma de tablas y gráficos. Posteriormente, se analizaron mediante estadística descriptiva con la ayuda del software IBM SPSS Statistics. El estudio fue aprobado por el Comité de Ética en Investigación. Resultados: La muestra, con una edad media de 55,5 \pm 5,0 años (27\%), fue mayoritariamente masculina $(58,3 \%)$; con educación primaria completa $(37,4 \%)$; renta familiar media de hasta 1 salario mínimo (44,3\%). De estos, solo el $41,7 \%$ de las personas eran conscientes de la importancia del calendario de vacunación. Además, el $86,1 \%$ afirmó creer que las vacunas son buenas, en contraste con el $90,4 \%$ que mostró tarjetas de vacuna obsoletas. Conclusión: Se concluye que existe un amplio espacio entre lo ideal y lo real, en lo que respecta a las prácticas de salud con fines de vacunación y el asiduo cumplimiento de la cartilla de vacunación, destacando su importancia y los problemas relacionados con la ausencia de esta prevención.

Palabras clave: Vacunación, Programa de inmunizacion, Cobertura de Vacunación.

\section{INTRODUÇÃO}

Luiz Pasteur, no século XIX, descobriu a possibilidade de produzir vacinas com microrganismos, bactérias e vírus (COSTA PG e VAZ LB, 2018). Posteriormente, estudos foram realizados e comprovou, assim, que a administração de imunobiológico confere imunização ativa ou passiva ao indivíduo e, com o passar dos anos, novas vacinas foram descobertas (MINISTÉRIO DA SAÚDE, 2014).

A partir do surgimento das vacinas, foi possível o desenvolvimento da medicina preventiva. Logo, esse feito permitiu o aumento significativo do espectro de estudo dento do âmbito da patologia. Em se tratando de doença, a ideia principal deixou de ser "como tratá-la?" e passou a ser, para muitos, "como evitá-la?" (HOCHMAN G, 2011). O processo de imunização das pessoas por meio de antígenos advindos de microrganismos patogênicos, revolucionou o cenário da época, transformando paradigmas, e proporcionou a proteção de milhares de pessoas. Graças a nossa memória imunológica, somos capazes de responder a uma segunda infecção de forma mais rápida, mais efetiva e com um grande título de anticorpos (ABBAS AK, et al., 2015).

O aumento da quantidade de pessoas que demonstraram preocupações acerca da segurança e efetividade das vacinas, veio juntamente com o aumento do número de vacinas disponíveis e seu uso por programas públicos de saúde e, por um momento, esse pareceu o caminho, com impacto comprovadamente positivo, mas, hoje, infelizmente, as frequentes epidemias por doenças imunopreveníveis ao redor do mundo colocam essa ideia em xeque (MIZUTA AH, et al., 2019).

O processo vacinal está intimamente relacionado a questionamentos e críticas sobre efeitos adversos, apesar da relevância já demonstrada na erradicação ou controle de muitas doenças infectocontagiosas. Além disso, existem eventos negativos envolvendo a indústria farmacêutica, como erros na produção que acabaram por afetar negativamente pessoas que optaram pela vacinação (APS LRMM, et al., 2018). Nesse sentido, cabe avaliar que muitas vezes a falta de informação sobre a importância da vacina e sobre as consequências da não vacinação, interfere diretamente na saúde coletiva (MIZUTA AH, et al., 2019).

A Organização Mundial da Saúde (OMS) recomenda que se realize 19 vacinas, com esquemas descritos pelo calendário nacional de vacinação, todas elas disponibilizadas pelo Sistema único de Saúde (SUS) (OMS, 2020). Assim, indaga-se sobre as causas que levam as pessoas a não se vacinarem. Elas sabem da importância da imunização? Frequentemente, questionamentos e críticas voltam olhares para os projetos de vacinação em massa, aumentando a hesitação vacinal e implicando em consequências para a saúde coletiva (APS LRMM, et al., 2018). 
É certo que os investimentos governamentais cada vez maiores no Programa Nacional de Imunização visam manter altos níveis de cobertura vacinal para população. Contudo, devido ao aumento do número de doses aplicadas e, respectivamente, do maior risco de efeitos adversos, o conflito ético entre a autonomia individual e o bem coletivo é atenuado. Afinal, seria a obrigatoriedade da vacinação uma violação da autonomia e vulnerabilização do sujeito quanto à sua individualidade? Ou seria o Estado promovendo conscientização da população e controle sanitário, a fim de evitar riscos epidêmicos? Logo, a existência de uma possível "cultura de imunização" no Brasil, trouxe mais benefícios ou malefícios para a aplicabilidade do calendário vacinal ao cidadão brasileiro? (JESUS AS, et al., 2016).

Por outro lado, porém, a escassez de orientação populacional mostra-se um obstáculo, o que gera a problemática negligência da medicina preventiva. É responsabilidade dos profissionais de saúde fornecer informações para seus pacientes a respeito da vacinação correta, de acordo com as evidências científicas mais atualizadas e recomendadas. Dessa forma, faz-se necessário o desenvolvimento de estratégias de ensino continuado e treinamento para garantir que os profissionais da saúde aprimorem seu conhecimento e habilidade a respeito do tema. Tais estratégias teriam como objetivo causar um impacto positivo na conscientização da população e na cobertura vacinal em áreas de baixa adesão aos programas de imunização (PELULLO CP, et al., 2020).

As vacinas são um investimento em saúde com impacto positivo comprovado, uma vez que evitam milhões de mortes por ano e aumentam a expectativa e a qualidade de vida da população (MIZUTA AH, et al., 2019). O calendário vacinal abrange do lactente ao idoso, sendo de extrema importância mantê-lo sempre atualizado. Contudo, sabe-se que acreditar que há o cumprimento exato do cartão de vacina ainda é utópico a nível de Brasil (PINTO JUNIOR VL, et al., 2019).

Embora a medicina preventiva tenha vencido muitas barreiras nos últimos anos, ela ainda não possui aceitação universal. A hesitação vacinal tem como base, em sua maioria, ideias infundadas teoricamente e a própria ignorância sobre o assunto (PINTO JUNIOR VL, et al., 2019). Nesse passo, as consequências são, infelizmente, o aumento da frequência de epidemias de doenças imunopreveníveis. Frente a isso, fica claro a necessidade de, mesmo que em grupo específicos, atuar no levantamento de causas para a não vacinação e na proposta de conscientização sobre de seus benefícios (SATO APS, 2018).

Portanto, pesquisas nesse sentido são de extrema relevância e potencial impacto positivo. Dúvidas e incertezas quanto a segurança, a importância e o cronograma de vacinação existem, o que abre espaço para pesquisas como essa, que buscam identificar pontos de fraqueza do Sistema de Imunização, informar a comunidade científica e trazer um recado de conscientização para o grupo amostral. Diante do exposto, o presente estudo teve como objetivo analisar o cumprimento ou não do Calendário Vacinal, compreender a perspectiva de uma amostra acerca da prática da vacinação e concluir implicações disso, diretamente observadas, na Saúde Coletiva.

\section{MÉTODOS}

Foi realizado um estudo transversal descritivo de abordagem quantitativa, tendo como local de coleta de dados de uma clínica nefrológica. A população estudada foi de pacientes renais crônicos atendidos dos meses de março a agosto do ano de 2021. O grupo amostral do trabalho consiste em 115 indivíduos atendendo os critérios de inclusão. A coleta dos dados foi feita por meio de um questionário impresso, padrão, de autoria do grupo.

As características avaliadas para o estudo foram: idade, escolaridade, renda familiar média, sexo, orientação prévia sobre o calendário de imunização, percepção acerca dos benefícios ou malefícios da vacina, cumprimento do cartão de vacina, efeitos colaterais relacionados com vacinação, conhecimento científico sobre o processo vacinal e preenchimento do cartão em relação aos filhos.

Os pacientes que tiveram horário agendado para os dias de aplicação da pesquisa foram instruídos por nós do grupo do que se tratava e, os que aceitaram participar e consentiram com o Termo de Consentimento Livre e Esclarecido (TCLE), responderam, na nossa presença, o questionário. $O$ tempo para que o questionário fosse respondido foi de, em média, 10 minutos por paciente. 
Foram definidos como critérios de inclusão pacientes atendidos na Clínica de Nefrologia, com idade acima de 18 anos. Como critérios de exclusão, foram constatados pacientes sem condições de responder o questionário; pacientes que não preencheram o TCLE adequadamente e pacientes que vierem a óbito durante o estudo.

Os dados obtidos foram transcritos para uma planilha em Programa Microsoft (MS) Excel Office XP para análise estatística descritiva. Foram analisados com auxílio do software Internacional Business Machines (IBM) Statistical Package for the Social Science (SPSS) Statistics.

O projeto foi descrito e submetido ao Comitê de Ética em Pesquisa (CEP). A aprovação por esse órgão tem como CAAE: 40145020.4.0000.5076 e Número do Parecer 4.515.420/2021. Além disso, foi assinado um TCLE por parte dos participantes.

\section{RESULTADOS}

Ao fim da aplicação de questionários, realizada na Clínica de Nefrologia, em Aparecida de Goiânia, alcançamos um grupo amostral de 115 pacientes. Em se tratando do perfil socioeconômico dos referidos pacientes, concluiu-se que a maior parte é do sexo masculino (58,3\%); tem escolaridade até o 1 grau completo $(37,4 \%)$; faixa etária entre 51 e 60 anos (27\%); renda familiar média, majoritariamente, de até um saláriomínimo (44,3\%) e são, em sua totalidade, brasileiros (Tabela 1).

Tabela 1 - Distribuição dos casos de acordo com o perfil sociodemográfico da população do estudo ( $n=115)$.

\begin{tabular}{lcc}
\hline VARIÁVEIS & $\mathbf{n}$ & $\%$ \\
\hline Sexo & & 48 \\
\hline Feminino & 67 & 58,3 \\
Masculino & & 7 \\
\hline Faixa etária & 8 & 10,4 \\
\hline $18-30$ & 12 & 23,5 \\
$31-40$ & 27 & 27 \\
$41-50$ & 31 & 32,2 \\
51 - 60 & 37 & \\
$>60$ & & 27,9 \\
\hline Escolaridade & 32 & 37,4 \\
\hline Ensino Fundamental Incompleto & 43 & 24,3 \\
Ensino Fundamental Completo & 28 & 10,4 \\
Ensino Médio Completo & 12 & 44,3 \\
Ensino Superior Completo & & 32,2 \\
\hline Renda Familiar Média & 51 & 15,6 \\
\hline Até 1 salário-mínimo & 37 & 7,8 \\
1 -2 salários & 18 & \\
2-3 salários & 9 & \\
mais que 3 salários & & \\
\hline
\end{tabular}

Fonte: Loyola CF, et al., 2021.

No que tange o conhecimento e as perspectivas dos pacientes frente a vacinação e aos calendários vacinais, como constatado na Tabela 2, observa-se que a maior parcela dos pacientes $(58,3 \%)$ não foi informada sobre a importância do calendário de imunização, contudo grande parte $(52,2 \%)$ alegou saber para que serve a vacina. Além disso, constatou-se que $86,1 \%$ dos entrevistados consideram que as vacinas não fazem mal e 23,5\% alegaram já terem tido efeitos colaterais à vacina. Quanto ao cartão vacinal, 90,4\% deles estão desatualizados, 76,5\% afirmaram já terem tido mais de um cartão e, 76,5\% do grupo amostral alegam terem filhos e terem os levado para vacinar (Tabela 2). 
Tabela 2 - Conhecimento e perspectivas dos pacientes renais crônicos da clínica nefrológica, em relação ao calendário de imunização e a importância da vacinação $(n=115)$.

\begin{tabular}{lcc}
\hline VARIÁVEIS & $\mathbf{n}$ & $\%$ \\
\hline Já foi orientado sobre calendário de imunização? & & 36,5 \\
\hline Sim & 67 & 58,3 \\
Não & 6 & 5,2 \\
Não lembram & 16 & 13,9 \\
\hline Acham que vacina faz mal? & 99 & 86,1 \\
\hline Sim & & \\
Não & 11 & 9,6 \\
\hline Cartão está atualizado ou desatualizado? & 104 & 90,4 \\
\hline Atualizado & & \\
Desatualizado & 27 & 23,5 \\
\hline Já tiveram efeito colateral? & 88 & 76,5 \\
\hline Sim & & 52,2 \\
Não & 60 \\
\hline Sabe para que serve a vacina? & 27 & 23,5 \\
\hline Sim & 28 & 24,3 \\
Não & & 76,5 \\
Acha que sim & 88 \\
\hline Já teve mais de um cartão de vacina? & 22 \\
\hline Sim & 5 & 19,1 \\
Não & & 4,3 \\
Não sabe & 88 \\
\hline Tem filhos? Se sim, já levou para vacinar? & 2 & 76,5 \\
\hline Sim, e levei para vacinar & 5 & 1,7 \\
Sim, levei algumas vezes para vacinar & 20 & 4,3 \\
Sim, mas nunca levei para vacinar & & 17,4 \\
Não tenho filhos & &
\end{tabular}

Fonte: Loyola CF, et al., 2021.

Dentre os $23,5 \%$ dos pacientes que referiram terem tido efeitos colaterais à vacinação, $47,7 \%$ alegaram cefaleia e $36,8 \%$ alegaram febre (Figura 1). Avaliando, ainda, esse mesmo grupo amostral que relatou efeitos colaterais, apenas $5,2 \%$ deles eram contra a vacinação.

Figura 1 - Porcentagem de pessoas que apresentaram sintomas à vacinação.

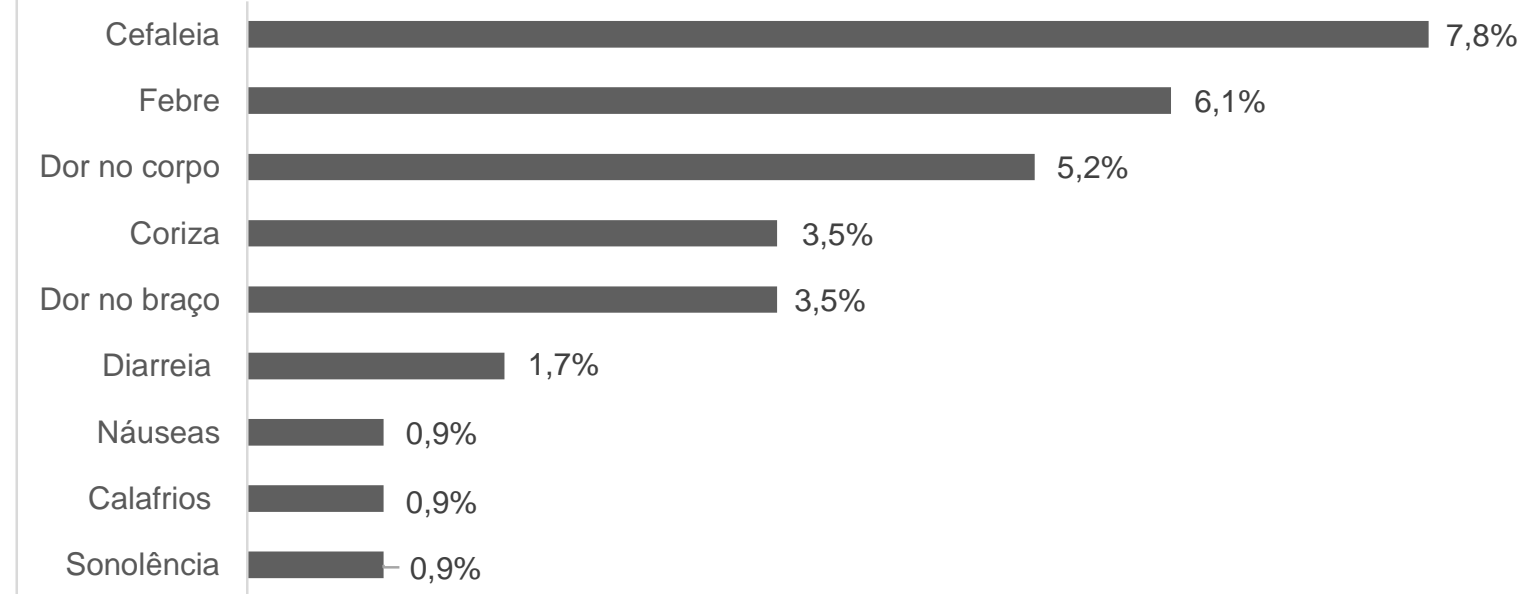

Fonte: Loyola CF, et al., 2021. 
Por outro lado, 8,7\% dos pacientes, mesmo não tendo relatado nenhum efeito colateral, também se mostraram contra a vacinação (Figura 2).

Figura 2 - Correlação entre efeitos colaterais e a perspectiva sobre o benefício ou malefício das vacinas.

Não tiveram efeito colateral e não acham que faz mal $67,8 \%$

Tiveram efeito colateral e não acham que faz mal

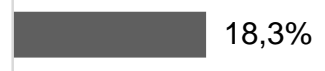

Não tiveram efeito colateral, mas acham que faz mal $8,7 \%$

Tiveram efeito colateral e acham que faz mal $5,2 \%$

Fonte: Loyola CF, et al., 2021.

\section{DISCUSSÃO}

A imunização tem se mostrado o principal meio de profilaxia de doenças imunopreviníveis. Tal fato está relacionado com seus resultados satisfatórios, em termos de custo e efetividade e, também por esses motivos, o processo vacinal ganhou espaço, tornando-se um componente obrigatório nos manuais e projetos de saúde pública através do Programa Nacional de Imunização (PNI). Entretanto, nota-se reaparecimento de patologias antes notificadas e ditas erradicadas e, ainda, surgimento de novas doenças, como a Pandemia da COVID19. Dessa forma, constatou-se em um estudo responsável por avaliar a cobertura vacinal no Brasil nos últimos 10 anos que a meta da imunização no país está cada vez mais longe de ser alcançada, uma vez que a cobertura vacinal reduziu ao longo desses anos (SILVA MCT, et al., 2020).

Por meio deste estudo, observou-se que mais da metade do grupo entrevistados não recebeu orientações acerca do calendário nacional de imunização em nenhum momento de suas vidas. Essa falta de informação impacta, negativamente, a sociedade de forma direta, tendo em vista que a vacina é uma das estratégias mais efetivas na prevenção e na redução da incidência de doenças infecciosas. Assim, percebe-se a necessidade de um maior direcionamento e uma maior fonte de informação para que ocorre uma consolidação do conhecimento científico em detrimento da informação advinda do imaginário popular (MINISTÉRIO DA SAÚDE, 2014).

No presente estudo, temos a cobertura vacinal como grande paradigma na garantia de impacto positivo na saúde coletiva. Logo, a vacinação de rotina consiste na implementação do Calendário Nacional de Vacinações, devendo ser aplicado a cada cidadão a partir dos seus primeiros dias de vida, objetivando garantir, no contexto individual, a prevenção específica de doenças imunopreviníveis e, no contexto coletivo, a estimulação da imunidade em massa, a fim de interromper a transmissão e contágio (MORAES JCD, et al., 2003).

Nesse passo, nota-se um gargalo no âmbito da saúde coletiva, uma vez que a cobertura vacinal ainda não é efetiva, na prática, de modo a gerar um impacto social. De acordo com a Organização Panamericana de Saúde (OPAS) (2019), recomenda-se a cobertura de 95\% da população, para que esta esteja imunizada de acordo com cada agente imunobiológico. O abandono e a desinformação acerca da importância de todas as doses que compõem os esquemas vacinais é fator importante para a diminuição da cobertura, fazendo com que o número de casos de específica doença aumente, provocando surtos no país, já que a imunização completa só é alcançada a partir da adesão de todas as doses estipuladas (SILVA JUNIOR JB, et al., 2013).

Este estudo tem o intuito de vincular essa falta de cobertura a um imaginário social que acredita que o processo vacinal esteja diretamente vinculado a algum malefício. Segundo Silva MCT, et al. (2020), os eventos adversos pós vacinação demonstraram queda em comparação com os primeiros anos disponíveis pelo Ministério da Saúde, sendo esse um dos principais motivos da resistência ao processo de vacinação. 
Nesse sentido, quando os entrevistados foram questionados sobre uma possibilidade de malefício proveniente das vacinas, a maioria dos entrevistados alegaram compreender o propósito da imunização e afirmaram que as vacinas, sob suas óticas, não causam prejuízos para a saúde.

Assim, infere-se que a presença de entendimento e compreensão por parte dos entrevistados sobre a eficácia preventiva das vacinas, interfere diretamente em suas condutas pessoais no que tange a aplicação do calendário vacinal e o cumprimento das datas. Nesse passo, mesmo não tendo uma avaliação precisa da proporção desse entendimento, evidenciou-se sua importância e suas repercussões para a saúde coletiva.

Quando perguntados sobre a função das vacinas, a maioria do grupo amostral relatou possuir entendimento, justificando suas respostas com a ideia da utilização vacinal para combater uma doença já instalada ou para a prevenção de uma doença ainda não contraída. Contudo, ainda que a maior parcela dos entrevistados relatasse entendimento, a diminuição percentual da vacinação a partir do ano de 2016 segue sendo uma verdade inquestionável (SATO APS, 2018).

Porém, por vezes, quando os entrevistados falavam sobre esse aspecto, percebeu-se que alguns deles, pela expressão facial e pelo tom de voz, carregavam certa insegurança ao discorrer, embora todos tivessem uma opinião formada e não omitissem a resposta. Nesse caso, no que tange a hesitação vacinal, motivos como vacinas com esquemas de mais de uma dose foram elencados como causas do abandono do esquema vacinal, repercutindo, consequentemente, na diminuição da imunização (SATO APS, 2018).

Todavia, uma boa parte dos entrevistados eram idosos e a eficácia da vacina nesses pacientes também é diferente. O próprio processo de envelhecimento gera redução das funções fisiológicas e a redução das soluções imunes do corpo para eventos agressivos pode levar a desequilíbrios na homeostase e aumentar a ameaça progressiva de patologia, que pode levar à morte individual. É importante notar que essas mudanças são provenientes de uma variedade de motivos, incluindo externos e internos (NUNES MI, et al.,2012).

Tendo por base o estudo realizado por Gomes LM, et al. (2012), vimos uma concordância com nossa pesquisa, já que o principal fator que leva um idoso a não se vacinar é a crença de que os efeitos colaterais em idosos são mais intensos e mais desastrosos do que a não vacinação, gerando uma enorme resistência e uma dificuldade na execução de campanhas.

Quando se tratava da vacinação dos próprios pacientes a resposta era uma, mas a comprovação era outra, pois ao mesmo tempo que afirmavam ser a favor das vacinas a maioria dos cartões se encontravam incompletos. No entanto, quando perguntávamos sobre filhos a maioria, que afirmavam ter filhos, mantiveram os cartões de vacina dos filhos completos. De acordo com Santos LB, et al. (2011), um grupo muito importante e que tende a receber uma quantidade maior de informações sobre vacinação é o grupo das gestantes. Como o pré-natal tem crescido cada vez mais para garantir a saúde tanto da mãe quanto da criança, as informações vacinais para esse grupo acabam sendo mais eficazes. De acordo com seu estudo, mães com idade de 1930 anos, trabalhadoras do lar, tinham um resultado melhor, já que tinham mais tempo para se dedicar à saúde de seus filhos, mesmo que a maioria também tivesse apenas o ensino fundamental completo.

Dessa forma, percebe-se que ainda existe uma fragilidade na construção da conscientização nacional maciça no que se refere ao calendário de imunização e à importância vacinal. Esse aspecto se torna um gargalo na saúde coletiva já que, no Brasil, a garantia do controle das doenças imunopreviníveis baseia-se, principalmente, na eficácia da cobertura vacinal (SANTOS LB, et al., 2011).

Reduzir e controlar o surgimento e a perpetuação de doenças e, com isso, diminuir as consequências atreladas a elas, é o principal objetivo da vacinação. Porém, para que essas metas sejam atingidas, faz-se necessário à adoção de uma série de cuidados em torno da administração desses imunobiológicos (MIZUTA $\mathrm{AH}$, et al., 2019).

Quando questionados se a vacina podia trazer riscos, mais da metade respondeu de forma objetiva que "não". De acordo com o estudo de Silva MN e Flauzino RF (2017), evento adverso pós-vacinação é qualquer intercorrência clínica indesejável em um indivíduo que tenha recebido a vacina, independentemente de estar ou não associado à vacina. 
Menos da metade do grupo amostral afirmaram ter apresentado eventos adversos, sendo os principais cefaleia, febre e dor no corpo. São eventos sistêmicos leves, que evoluem e desaparecem naturalmente, podendo ser tratados apenas com sintomáticos (SILVA MN e FLAUZINO RF, 2017).

De acordo com o manual de vigilância epidemiológica de eventos adversos pós vacinação apesar da confiabilidade das vacinas, estas podem provocar alguns efeitos adversos, podendo ser mais leves como febre, dor e edema local, ou mais graves como convulsões febris e choque anafilático. Porém o risco de ocorrer uma complicação mais grave é mínimo, frente aos benefícios proporcionados pela vacina e aos riscos de complicações pelas doenças imunopreveníveis (MINISTÉRIO DA SAÚDE, 2020).

Os efeitos nocivos associados ao uso de vacinas, se existem, são comprovados cientificamente, ocorrem com uma frequência muito baixa e são insignificantes em comparação com os riscos associados à não vacinação (SILVA MN e FLAUZINO RF, 2017). Estratégias de incentivo ao uso de vacinas têm sido tradicionalmente adotadas na saúde pública, mas podem não ser suficientes para garantir o aumento da cobertura vacinal. Nesse caso, é necessário manter um entendimento claro do valor das vacinas na população e entre os profissionais de saúde (MORAIS SLA, et al., 2018).

Por isso, a população deve receber informações amplas e acessíveis sobre os possíveis efeitos adversos da vacinação, visando o não comprometimento da confiança populacional acerca das vacinas e, consequentemente, uma baixa adesão vacinal, podendo implicar, a longo prazo, maiores complicações para a saúde coletiva (SILVA MN e FLAUZINO RF, 2017).

Os dados referentes à atualização do calendário vacinal nos mostraram que a maior parte dos entrevistados apresentavam cartão de vacina desatualizado e possuíam mais de um cartão, uma vez que sempre recebiam um novo registro quando iam se vacinar.

A grande maioria dos estudos nos mostra o cumprimento do calendário vacinal em crianças e adolescentes. Quando encontrados estudos relacionados ao status do cartão de vacinas em adultos, a maioria se tratava de obrigatoriedade de atualização por conta do emprego. De acordo com Gonçalves AA (2019), em seu estudo sobre o desafio para vacinar adultos em uma Unidade Básica de Saúde (UBS) de Porto Velho, podemos confirmar a crença de vacinação como algo apenas para crianças, já que a maioria dos adultos estavam ali para acompanhar as crianças e que os idosos realmente acreditam que os efeitos adversos são piores do que a não vacinação.

Objetivando uma cobertura vacinal eficiente, é necessário evitar que oportunidades de vacinação sejam perdidas. Para tanto, os profissionais de saúde devem estar atentos e capacitados para identificar indivíduos que não estejam com o calendário vacinal atualizado, perguntado diretamente ou para os responsáveis e verificando o Cartão de imunização ou outro documento que tenha o registro da aplicação das vacinas (SATO APS, 2018).

\section{CONCLUSÃO}

Baseado nos dados obtidos, evidenciou-se que a maioria dos entrevistados sabem da importância da vacinação e não consideram o processo vacinal como um fator de risco para a saúde. Entretanto, apesar dos pacientes saberem de tal importância, notou-se que esse conhecimento é superficial, baseado no senso comum e sem orientação prévia adequada pelos profissionais de saúde, justificando, assim, a baixa adesão ao plano de imunização. Dessa forma, o grande motivo dos pacientes não estarem se vacinando, não estaria pautado no medo das reações adversas que a vacina poderia provocar, mas, sim, na falta de informação adequada sobre os reais impactos positivos da vacinação na saúde e na relevância da prevenção para uma qualidade de vida plena. Logo, urge-se a necessidade de a equipe médica, em geral, reforçar e orientar adequadamente sobre a importância da vacinação e da completude do cartão vacinal em toda e qualquer oportunidade de contato com o paciente, evidenciando sua magnitude e aumentando, por fim, a adesão da população brasileira ao Programa Nacional de Imunização. 


\section{REFERÊNCIAS}

1. ABBAS AK, et al. Imunologia celular e molecular. 8aㅗ ed. Rio de Janeiro: Editora Ltda., 2015; 1-11p.

2. APS LRMM, et al. Eventos adversos de vacinas e as consequências da não vacinação: uma análise crítica. Revista de Saúde Pública, 2018; 52: 40.

3. ARAÚJO TM, et al. Vacinação e fatores associados entre trabalhadores da saúde. Cadernos de Saúde Pública, 2019; 35: e00169618.

4. COSTA PG, VAZ LB. A descoberta da vacina: uma história de sucesso no combate a grandes epidemias. Faculdade de Atenas, 2009.

5. GOMES LM, et al. Motivos que levaram os idosos a não se vacinarem contra a influenza sazonal. Ed. Jul./Set. Revista de pesquisa: Cuidado é fundamental online, 2012.

6. GONÇALVES AA. O desafio de vacinar adultos em uma unidade básica de saúde de porto velho. São Lucas Educacional, 2019.

7. HOCHMAN G. Vacinação, varíola e uma cultura da imunização no Brasil. Ciência \& Saúde Coletiva, 2011; 16(2): 375386.

8. MINISTÉRIO DA SAÚDE. Secretaria de Vigilância em saúde. Departamento de vigilância das doenças transmissíveis. Manual de normas e procedimentos para vacinação. Brasil, 2014. Disponível em: https://bvsms.saude.gov.br/bvs/publicacoes/manual_procedimentos_vacinacao.pdf.Acessado em 29 de Novembro de 2021.

9. MINISTÉRIO DA SAÚDE. Secretaria de Vigilância em saúde. Departamento de vigilância das doenças transmissíveis. Manual de vigilância epidemiológica de eventos adversos pós-vacinação. Brasil, 2020. Disponível em: https://bvsms.saude.gov.br/bvs/publicacoes/manual_vigilancia_epidemiologica_eventos_adversos_pos_vacinacao.pd f. Acessado em: 29 de Novembro de 2021.

10. MIZUTA, AH, et al. Percepções acerca da importância das vacinas e da recusa vacinal numa escola de medicina. Revista Paulista de Pediatria, 2019; 37(1): 34-40.

11. MORAES, JCD, et al. Qual é a cobertura vacinal real? What is the real vaccinattion coverage? Epidemiologia e Serviços de Saúde, 2003; 12(3).

12. MORAIS, SLA, et al. A evolução histórica do calendário vacinal brasileiro infantil. Enfermagem Atual InDerme, 2018; $85(23)$.

13. MOULíN AM. A hipótese vacinal: por uma abordagem crítica e antropológica de um fenômeno histórico. História, Ciências, Saúde-Manguinhos, 2003; 10: 499-517.

14. NUNES MI, et al. Enfermagem em Geriatria e Gerontologia. Editoração Eletronica. Rio de Janeiro: Guanabara Koogan, $2012 ; 2(10)$.

15. ORGANIZAÇÃO PAN-AMERICANA DE SAÚDE (OPAS). Brasil, 2020. Disponível em: https://www.paho.org/pt/covid19. Acessado em: 2 de Dezembro de 2021.

16. PELULLO CP, et al. Healthcare Workers' Knowledge, Attitudes, and Practices about Vaccinations: A Cross-Sectional Study in Italy. Vaccines, 2020; 8(2): 148.

17. PINTO JUNIOR VL. Anti-vacinação, um movimento com várias faces e consequências. Cadernos Ibero-Americanos de Direito Sanitário, 2019; 8(2): 116-122.

18. SANTOS LB, et al. Percepção das mães quanto à importância da imunização infantil. Revista Rene, Fortaleza, 2011; 12(3): 621-6.

19. SATO APS. Qual a importância da hesitação vacinal na queda das coberturas vacinais no Brasil?. Revista de Saúde Pública, 2018; 52.

20. SILVA JUNIOR JB, et al. 40 anos do Programa Nacional de Imunizações: uma conquista da Saúde Pública brasileira. Epidemiologia e Serviços de Saúde, 2013; 22(1): 7-8.

21. SILVA MN, FLAUZINO RF. Rede de frio: gestão, especificidades e atividades [online]. Rio de Janeiro: CDEAD/ENSP/EPSJV/Editora FIOCRUZ, 2017, $348 \mathrm{p}$.

22. SILVA JIB, et al. Adesão a Imunização entre Idosos. In: Congresso Internacional de Enfermagem. 2017.

23. SILVA MCT, et al. Estudo da cobertura vacinal no Brasil nos últimos 10 anos. / Mariana Campideli Teixeira Silva. Fundação Educacional Vale do São Francisco - FEVASF-MG. Iguatama, 2020. 